\title{
'|||||||||||||||||||||||||||||||||||||||||||||||||||||||. Connected Agricultural Machinery
}

\section{Dear Reader,}

Agricultural machinery will undergo lasting changes over the next few years as a result of increasing digitisation and connectivity. This will be evident at Agritechnica, which will take place from 12 to 18 November 2017 in Hanover, Germany. Dr. Bernd Scherer from VDMA defines the term digitisation in our interview on page 18 as "the web-based connection of the agricultural production process along the entire value chain". Communication between machines is therefore state of the art today. In the future, the aim will be to extend the value chain right through to the supermarket shelf, making it possible to trace a product back to its origins.

Once again this year, the diesel engine will be the dominant source of driving power at Agritechnica - and this will not change at least in the medium term. Agricultural machinery manufacturers are, however, looking for alternative solutions. For example, Agco Fendt is presenting its Vario e100 battery-powered tractor at Agritechnica 2017, while John Deere showed a battery-powered concept study at SIMA 2017. But engineers searching for alternative powertrain solutions should definitely keep an open mind with regard to technologies. For that reason, research and development should continue to work on hybrid solutions, alternative fuels and, not least, the fuel cell. After all, what is often neglected in the discussion concerning electric drive systems is the issue of availability. Where does the electricity that I need for charging the battery come from and how do I create the necessary infrastructure?
After three lean years, the agricultural machinery business is now picking up again. This fact, along with the broad range of innovations on display, is likely to attract large numbers of visitors to Hanover once again. For example, the German Agricultural Society (DLG) has received more than 320 announcements of innovations from a total of 175 exhibitors from 24 countries. I hope to see as many of these as possible live when I visit the show and I look forward to expanding my personal network.

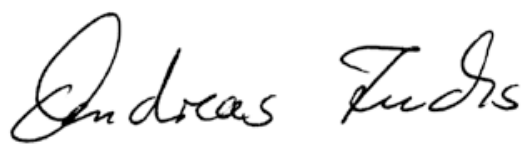

Andreas Fuchs

Chief Correspondent

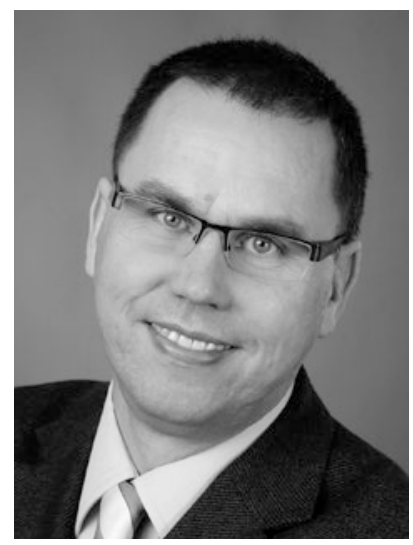

